\title{
Buoyancy and capillary effects on floating liquid lenses
}

\author{
P. D. Ravazzoli $\odot,{ }^{1,}{ }^{*}$ A. G. González $\odot,{ }^{1}$ J. A. Diez $\odot,{ }^{1}$ and H. A. Stone ${ }^{2}$ \\ ${ }^{1}$ Instituto de Física Arroyo Seco, Universidad Nacional del Centro de la Provincia de Buenos Aires, \\ and CIFICEN-CONICET-CICPBA, Pinto 399, 7000 Tandil, Argentina \\ ${ }^{2}$ Department of Mechanical and Aerospace Engineering, Princeton University, Princeton, \\ New Jersey 08544, USA
}

(Received 21 April 2020; accepted 9 July 2020; published 24 July 2020)

\begin{abstract}
We study the equilibrium shape of a liquid drop resting on top of a liquid surface, i.e., a floating lens. We consider the surface tension forces in nonwetting situations (negative spreading factor), as well as the effects of gravity. We obtain analytical expressions for the drop shape when gravity can be neglected. Perhaps surprisingly, when including gravity in the analysis, we find two different families of equilibrium solutions for the same set of physical parameters. These solutions correspond to drops whose center of mass is above or below the level of the external liquid surface. By means of energetic considerations, we determine the family that has the smallest energy, and therefore is the most probable to be found in nature. A detailed explanation of the geometrical differences between the two types of solutions is provided.
\end{abstract}

DOI: 10.1103/PhysRevFluids.5.073604

\section{INTRODUCTION}

Fluid-fluid interactions between two immiscible liquids are common in nature and in many industrial processes. Pioneering work goes back at least to Benjamin Franklin [1], but a plethora of papers have been devoted to the spreading phenomenon of one liquid over another (see, e.g., Lord Rayleigh [2], Neumann and Wangerin [3], Hardy [4], Lyons [5], Langmuir [6], Miller [7], Zisman [8], Seeto et al. [9], and Takamura et al. [10]).

In more recent years, the wettability of liquids over liquids has continued to be studied with a focus on new features. For example, Wyart et al. [11] studied liquid films dewetting from another liquid. Burton et al. [12], and more recently Tress et al. [13], analyzed the shape of a liquid lens, while Chen et al. [14] studied the dependence of the lens size on the contact angle, and McBride et al. [15], Endoh et al. [16], Levich et al. [17], and Sebilleau et al. [18] have been concerned with the spreading phenomenon.

Physically, a liquid lens is a drop lying over another immiscible liquid and surrounded by a gas phase, such as air. At equilibrium, the three phases meet along a circular line, where the sum of the three tensions must be zero. Neumann's rule [19,20] is the corresponding version of the Young equation for a solid substrate, and it provides the balance between the tensions at the contact line. Recently, several authors have addressed different aspects of floating lenses. The evaporation process of a liquid lens has been considered in [21] and compared with a theoretical model constructed assuming a constant contact angle and spherical cap shape. Also, the interaction, coalescence, and repulsion of floating drops were studied in [22] and [23].

Other authors have studied a similar geometrical configuration, namely a compound drop, where the liquid lens rests on a larger drop sitting on a solid substrate. In this case, there is not only the interaction of two liquid phases (lens and drop) with the ambient gas (air), but also the liquid drop

*Corresponding author: pravazzoli@ifas.exa.unicen.edu.ar 


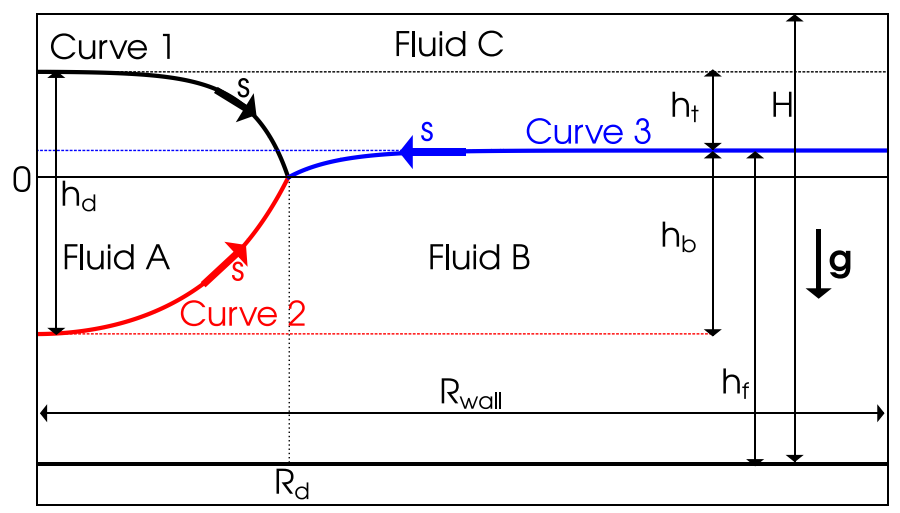

FIG. 1. Dimensionless scheme of a liquid lens (fluid $A$ ) over a deep external liquid (fluid $B$ ), surrounded by air (fluid $C$ ). The triple contact line is at $r=R_{d}$ and $z=0\left(R_{d} \ll R_{\text {wall }}\right)$. The arrows over each curve indicate the increasing direction of the arclength, $s$. The length scale is $\mathcal{R}_{0}$ [Eq. (1)].

with the solid. As in the liquid lens case, Neumann's rule plays an important role, but a larger number of free parameters is required to analyze all possible static states. An example of this type of study is that of Zhang et al. [24], where seven parameters were identified, even though they considered a limit case in which only four were relevant. Several aspects of the compound drop have been studied in the literature, such as merging of sessile drops [25,26] and its equilibrium shape [27].

To study the dynamic behavior of a liquid lens, it is necessary to achieve a full understanding of the static case and how the physical parameters affect the shape of the resulting drop. Even if the liquid lens shape has been studied previously by several authors [12,28-31], we present here some aspects of the static solutions that have not been fully addressed. In the present work, we initially follow the guidelines presented by Burton et al. [12] for the partial wetting situation, and numerically solve the three pairs of coupled differential equations resulting from the pressure balance on each surface.

In Sec. II, we present the basic formalism and define the appropriate dimensionless parameters to focus on surface tension effects. We characterize the problem by using certain sets of parameters, namely a reference Bond number (Bo), the ratios of the three surface tensions, and the dimensionless spreading factor. Then, we analytically solve the problem without considering gravity effects, and we obtain expressions for the two spherical caps that constitute an equilibrium floating drop.

In Sec. IV we take into account the gravity effects, and we identify the existence of two families of solutions for the same set of parameters. To the best of our knowledge, this interesting result has not been reported previously. Here, we show its existence and give a detailed description. To decide which type of solution is more likely to be found in nature, we perform an energetic analysis in Sec. V. We calculate the system energy for the different scenarios and find that one family of solutions is always lower in energy than the other.

\section{DESCRIPTION OF THE PROBLEM AND FORMALISM}

\section{A. Governing equations and boundary conditions}

We are interested in the shapes of static interfaces that develop when a drop is deposited on a liquid surface under the effects of both surface tension and gravity. In particular, we consider the case when a drop (fluid $A$ ) floats under partial wetting conditions on the liquid-air interface (fluids $B, C)$; see Fig. 1. To scale the problem, we use a characteristic length scale given by

$$
\mathcal{R}_{0}=\left(\frac{3 \mathcal{V}_{0}}{4 \pi}\right)^{1 / 3},
$$

where $\mathcal{V}_{0}$ is the volume of the drop. 
In this dimensionless configuration (see Fig. 1), we assume that the drop radius, $R_{d}$, is much smaller than the size of the container (of radius $R_{\text {wall }}$ ), and that the thickness of the lower layer, $h_{f}$, is always large enough to assure that the drop never touches the bottom regardless of the drop volume, $V=\mathcal{V}_{0} / \mathcal{R}_{0}^{3}$. The interface curves between each fluid are denoted by numbers. Thus, curve 1 corresponds to the interface between fluids $A$ and $C$, curve 2 to $A$ and $B$, and curve 3 to $B$ and $C$. As shown in Fig. 1, the arc length along each curve, $s$, increases from 0 toward the triple point, where the three curves meet.

Since surface tension forces are responsible for the Laplace pressure jump across the liquid interfaces, we can write

$$
p_{i}-p_{j}=\sigma \kappa,
$$

where $p_{i}$ and $p_{j}$ are the hydrostatic pressures in the bulk of each fluid at both sides of the corresponding curve of curvature $\kappa$, so that $i$ and $j$ stand for $A, B$, or $C$. Thus, the equilibrium equation for each interface can be written

$$
\begin{aligned}
& \left(P_{C}-P_{A}\right)+g z\left(\rho_{A}-\rho_{C}\right)=\sigma_{1} \kappa_{1}, \\
& \left(P_{A}-P_{B}\right)+g z\left(\rho_{B}-\rho_{A}\right)=\sigma_{2} \kappa_{2}, \\
& \left(P_{B}-P_{C}\right)+g z\left(\rho_{C}-\rho_{B}\right)=\sigma_{3} \kappa_{3},
\end{aligned}
$$

where $P$ refers to the reference pressure inside each fluid, and the subscripts in capital letters and numbers, respectively, correspond to a fluid and the interfaces between them. The dimensionless form of these equations can be written as

$$
\Delta P_{i}+\mathrm{Bo}_{i} z_{i}(s)=\kappa_{i}(s), \quad i=1,2,3,
$$

where all lengths are expressed in units of $\mathcal{R}_{0}$, and we have defined the dimensionless constants

$$
\begin{aligned}
\Delta P_{1} & =\frac{\mathcal{R}_{0}}{\sigma_{1}}\left(P_{C}-P_{A}\right), \\
\Delta P_{2} & =\frac{\mathcal{R}_{0}}{\sigma_{2}}\left(P_{A}-P_{B}\right), \\
\Delta P_{3} & =\frac{\mathcal{R}_{0}}{\sigma_{3}}\left(P_{B}-P_{C}\right), \\
\mathrm{Bo}_{1} & =\left(\frac{\rho_{A}-\rho_{C}}{\sigma_{1}}\right) g \mathcal{R}_{0}^{2}, \\
\mathrm{Bo}_{2} & =\left(\frac{\rho_{B}-\rho_{A}}{\sigma_{2}}\right) g \mathcal{R}_{0}^{2}, \\
\mathrm{Bo}_{3} & =\left(\frac{\rho_{C}-\rho_{B}}{\sigma_{3}}\right) g \mathcal{R}_{0}^{2} .
\end{aligned}
$$

Assuming axial symmetry for this problem, the dimensionless surface curvature is given by [12]

$$
\kappa_{i}=\frac{z_{i}^{\prime}(s)}{r_{i}(s)\left[r_{i}^{\prime}(s)^{2}+z_{i}^{\prime}(s)^{2}\right]^{1 / 2}}+\frac{r_{i}^{\prime}(s) z_{i}^{\prime \prime}(s)-z_{i}^{\prime}(s) r_{i}^{\prime \prime}(s)}{\left[r_{i}^{\prime}(s)^{2}+z_{i}^{\prime}(s)^{2}\right]^{3 / 2}},
$$

where a prime denotes the derivative with respect to $s$. If $L_{i}$ is the entire arc length of any of the curves $(1,2$, or 3$)$, then we scale the arc length as $q=s / L_{i}(0 \leqslant q \leqslant 1)$ so that

$$
\kappa_{i}=\frac{z_{i}^{\prime}(q)}{r_{i}(q) L_{i}}+\frac{r_{i}^{\prime}(q) z_{i}^{\prime \prime}(q)-z_{i}^{\prime}(q) r_{i}^{\prime \prime}(q)}{L_{i}^{3}},
$$


where

$$
L_{i}^{2}=r_{i}^{\prime}(q)^{2}+z_{i}^{\prime}(q)^{2}=\text { const. }
$$

This condition allows us to obtain two equations for $\left(r_{i}(q), z_{i}(q)\right)$ as

$$
\begin{aligned}
& r_{i}^{\prime \prime}(q)=z_{i}^{\prime}(q)\left[\frac{z_{i}^{\prime}(q)}{r_{i}(q)}-L_{i} \kappa_{i}\right], \\
& z_{i}^{\prime \prime}(q)=-r_{i}^{\prime}(q)\left[\frac{z_{i}^{\prime}(q)}{r_{i}(q)}-L_{i} \kappa_{i}\right] .
\end{aligned}
$$

By replacing here the curvatures from Eq. (4), we obtain the three pairs of equations for $\left(r_{i}(q), z_{i}(q)\right)$ along each interface $(i=1,2,3)$,

$$
\begin{aligned}
& r_{i}^{\prime \prime}(q)=z_{i}^{\prime}(q)\left[\frac{z_{i}^{\prime}(q)}{r_{i}(q)}-L_{i} \Delta P_{i}-L_{i} \mathrm{Bo}_{i} z_{i}(s)\right], \\
& z_{i}^{\prime \prime}(q)=-r_{i}^{\prime}(q)\left[\frac{z_{i}^{\prime}(q)}{r_{i}(q)}-L_{i} \Delta P_{i}-L_{i} \mathrm{Bo}_{i} z_{i}(s)\right] .
\end{aligned}
$$

The integration of all three curves starts with zero slopes at the corresponding $q=s / L=0$, and it ends at the contact point. At the beginning point $(q=0)$ we have $z_{1}^{\prime}=z_{2}^{\prime}=z_{3}^{\prime}=0$, and according to Eq. (8) it must be $r_{1}^{\prime}=L_{1}, r_{2}^{\prime}=L_{2}$, and $r_{3}^{\prime}=-L_{3}$, since $r$ increases along curves 1 and 2 and decreases along curve 3 . The three curves meet at the triple contact point at $q=1$, where $r_{i}(1)=R_{d}$ and $z_{i}(1)=0$. The condition on $z$ is arbitrary since the system is translationally invariant in this direction, since the gravitational potential is linear in $z$. Thus, we will use this property to start all three integrations from $z=0$, and we proceed to make the corresponding vertical displacements a posteriori.

Note that both $L_{i}$ and $\Delta P_{i}$ are not known a priori in Eqs. (10). These six constants and the drop radius, $R_{d}$, must be determined consistently by solving all six equations plus the conservation of drop volume. The first three conditions are

$$
r_{1}=r_{2}=r_{3}=R_{d}
$$

at $q=1$. The fourth and fifth constraints are related to the so-called Neumann's rule, i.e., that surface tension forces must equilibrate along both $r$ and $z$ directions (see Fig. 2),

$$
\begin{array}{r}
\sigma_{1} \cos \alpha+\sigma_{2} \cos \beta-\sigma_{3} \cos \gamma=0, \\
\sigma_{1} \sin \alpha-\sigma_{2} \sin \beta+\sigma_{3} \sin \gamma=0,
\end{array}
$$

at $q=1$. The sixth condition is concerned with the evaluation of the reference pressures, $P_{i}$. In fact, by summing up the three equations in Eq. (3) at $z=0$,

$$
\sigma_{1} \kappa_{1}+\sigma_{2} \kappa_{2}+\sigma_{3} \kappa_{3}=0
$$

at $q=1$. Finally, the seventh condition is of the integral type, since it refers to the constraint of a given drop volume. Thus, we have

$$
V=\frac{\mathcal{V}_{0}}{\mathcal{R}_{0}^{3}}=\int_{0}^{1} 2 \pi r_{1}(q) r_{1}^{\prime}(q) z_{1}(q) d q-\int_{0}^{1} 2 \pi r_{2}(q) r_{2}^{\prime}(q) z_{2}(q) d q=\frac{4 \pi}{3}
$$

Therefore, the seven conditions in Eqs. (11)-(14) determine the shapes of the interfaces as well as the values of $L_{i}, \Delta P_{i}$, and $R_{d}$. 


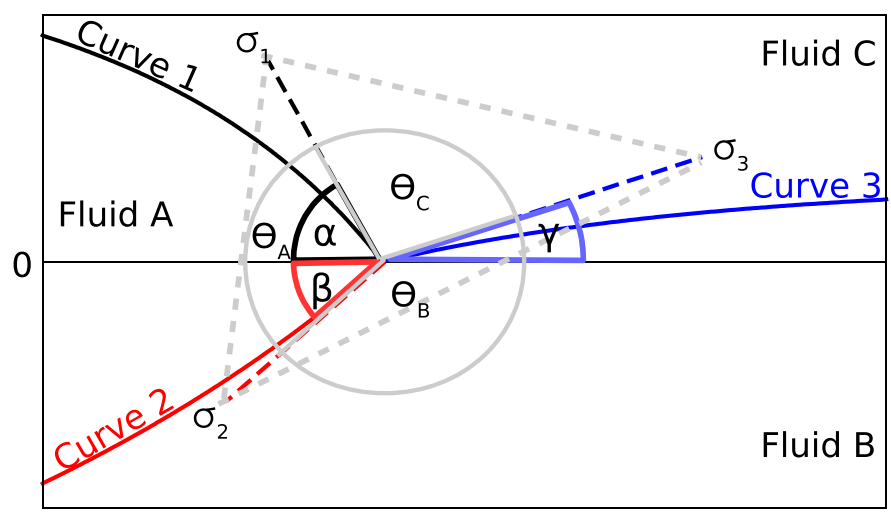

FIG. 2. Contact angle definitions for a liquid lens (fluid $A$ ) over a deep liquid substrate (fluid $B$ ), surrounded by air (fluid $C$ ).

A detailed analysis of the Neumann equilibrium conditions allows us to find relationships between the contact angles and the surface tensions. In fact, by using the cosine law in the triangle depicted in Fig. 2, we have

$$
\begin{aligned}
& \cos \theta_{A}=\frac{\sigma_{3}^{2}-\sigma_{2}^{2}-\sigma_{1}^{2}}{2 \sigma_{1} \sigma_{2}}, \\
& \cos \theta_{B}=\frac{\sigma_{1}^{2}-\sigma_{2}^{2}-\sigma_{3}^{2}}{2 \sigma_{2} \sigma_{3}}, \\
& \cos \theta_{C}=\frac{\sigma_{2}^{2}-\sigma_{1}^{2}-\sigma_{3}^{2}}{2 \sigma_{1} \sigma_{3}},
\end{aligned}
$$

which led to restrictions on the admissible values of the spreading coefficient,

$$
S=\sigma_{3}-\sigma_{2}-\sigma_{1},
$$

which is also used to describe the contact line motion on solid substrates. For convenience, we also write Eqs. (15) in terms of contact angles as (see Fig. 2)

$$
\alpha+\beta=\arccos \left(\frac{\sigma_{3}^{2}-\sigma_{2}^{2}-\sigma_{1}^{2}}{2 \sigma_{1} \sigma_{2}}\right), \quad \alpha+\gamma=\pi-\arccos \left(\frac{\sigma_{2}^{2}-\sigma_{1}^{2}-\sigma_{3}^{2}}{2 \sigma_{1} \sigma_{3}}\right) .
$$

Note that the knowledge of one angle and the three interfacial tensions automatically determines the other two.

From the fact that the moduli of Eqs. (15) must be less than 1, we find the following restrictions for the spreading coefficient:

$$
S<0, \quad-2 \sigma_{2}<S, \quad-2 \sigma_{1}<S,
$$

which can be summarized as

$$
-2 \min \left(\sigma_{1}, \sigma_{2}\right)<S<0 .
$$

Therefore, the condition for partial wetting (i.e., the formation of a static floating drop) is more restrictive than in the case of partial wetting of a solid substrate, which simply requires $S<0$.

\section{B. Nondimensionalization}

Since there are many dimensional parameters necessary to determine the final equilibrium solution, it is useful to define the problem in terms of a smaller number of dimensionless variables. 

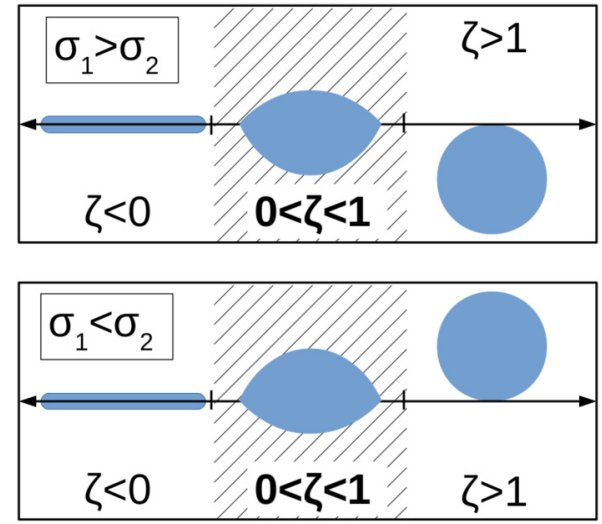

FIG. 3. Schematic of drop shapes for a given $\eta$ as a function of $\zeta$, with $\sigma_{1}>\sigma_{2}$ (top panel) and $\sigma_{1}<\sigma_{2}$ (bottom panel). The central dashed area where $0<\zeta<1\left(S^{*}<S<0\right)$ corresponds to the partial wetting scenario studied in this work. For $\zeta<0(S>0)$, we have complete wetting, so that the drop spreads indefinitely. Instead, for $\zeta>1\left(S<S^{*}\right)$ a nonwetting case occurs, where the drop remains on top or above the free surface, depending on the relative values of $\sigma_{1}$ and $\sigma_{2}$.

To develop this description, it is necessary to select reference values for both density and surface tension, namely $\rho_{\text {ref }}$ and $\sigma_{\text {ref }}$, respectively. For convenience we choose these values as $\rho_{\text {ref }}=\rho_{A}$ and $\sigma_{\text {ref }}=\min \left(\sigma_{1}, \sigma_{2}\right)$, where the latter selection is suggested by the condition in Eq. (19).

If we define the ratio

$$
\zeta=\frac{S}{S^{*}}, \quad S^{*}=-2 \sigma_{\mathrm{ref}},
$$

where $S^{*}$ is the reference spreading coefficient, all possible solutions correspond to the interval $0 \leqslant \zeta \leqslant 1$. So, from Eq. (16) we can write

$$
\zeta=\frac{1}{2}\left(\eta_{1}+\eta_{2}-\eta_{3}\right)
$$

where

$$
\eta_{1}=\frac{\sigma_{1}}{\sigma_{\text {ref }}}, \quad \eta_{2}=\frac{\sigma_{2}}{\sigma_{\text {ref }}}, \quad \text { and } \quad \eta_{3}=\frac{\sigma_{3}}{\sigma_{\text {ref }}} .
$$

Two scenarios are possible within this scheme:

(i) Case A $\left(\sigma_{1}<\sigma_{2}\right)$ :

$$
\eta_{1}=1, \quad \eta_{2}=\frac{\sigma_{2}}{\sigma_{1}} \equiv \eta>1, \quad \eta_{3}=\frac{\sigma_{3}}{\sigma_{1}}, \quad \zeta=\frac{1}{2}\left(1+\eta-\eta_{3}\right), \quad \sigma_{\text {ref }}=\sigma_{1} .
$$

(ii) Case $B\left(\sigma_{1}>\sigma_{2}\right)$ :

$$
\eta_{1}=\frac{\sigma_{1}}{\sigma_{2}} \equiv \eta>1, \quad \eta_{2}=1, \quad \eta_{3}=\frac{\sigma_{3}}{\sigma_{2}}, \quad \zeta=\frac{1}{2}\left(1+\eta-\eta_{3}\right), \quad \sigma_{\text {ref }}=\sigma_{2} .
$$

In the following, we use variables $\eta>1$ and $0<\zeta<1$ to treat both cases simultaneously, since these two variables are sufficient to include all possible values of surface tensions.

The different wetting possibilities given by Eq. (19) are schematically shown in Fig. 3 in terms of the dimensionless parameters $\eta$ and $\zeta$. The left column in the figure represents $S>0$, where the drop spreads over the liquid surface. Therefore, complete wetting occurs for both case $\mathrm{A}\left(\sigma_{1}<\sigma_{2}\right)$ and case $\mathrm{B}\left(\sigma_{1}>\sigma_{2}\right)$. The right column represents the nonwetting case, where the drop finds an equilibrium just below the interface for case B and just above for case A. Finally, in the center column, we have the partial wetting case, which is the scenario studied here. 
By using these definitions, we can rewrite the three equilibrium equations, Eq. (4), in dimensionless form as

$$
\begin{array}{r}
\Delta P_{1}+\left(\frac{\text { Bo }}{\eta_{1}}\right)\left(\frac{\rho_{A}-\rho_{C}}{\rho_{A}}\right) z=\kappa_{1}, \\
\Delta P_{2}+\left(\frac{\text { Bo }}{\eta_{2}}\right)\left(\frac{\rho_{B}-\rho_{A}}{\rho_{A}}\right) z=\kappa_{2}, \\
\Delta P_{3}+\frac{\text { Bo }}{\eta_{1}+\eta_{2}-2 \zeta}\left(\frac{\rho_{C}-\rho_{B}}{\rho_{A}}\right) z=\kappa_{3},
\end{array}
$$

where

$$
\text { Bo }=\frac{\rho_{A} g \mathcal{R}_{0}^{2}}{\sigma_{\text {ref }}}
$$

is the reference Bond number.

\section{ANALYTICAL SOLUTION WITHOUT GRAVITY}

As a first attempt to solve this problem, we consider the case without gravity. One feature of this simplification is that the pressure $p_{i}$ is only determined by the reference pressure in each bulk, $P_{i}$, due to the absence of the buoyancy contribution. This fact implies that the reference pressures $P_{B}$ and $P_{C}$ must be equal at both sides of curve 3 for all points along this curve, so that, $\kappa_{3}=0$ and curve 3 is completely flat. With this condition $\mathrm{Bo}=0$, we have two simplified equations to be solved on the drop surfaces,

$$
\Delta P_{i}=\kappa_{i} \quad \text { for } \quad i=1,2 .
$$

Since a flat curve 3 implies $\gamma=0$ (Fig. 2), Eq. (17) allows us to write $\alpha$ and $\beta$ in terms of the dimensionless parameters $\eta$ and $\zeta$ as

$$
\begin{aligned}
& \alpha=\pi-\arccos \left[\frac{\eta_{2}^{2}-\eta_{1}^{2}-\left(\eta_{1}+\eta_{2}-2 \zeta\right)^{2}}{2 \eta_{1}\left(\eta_{1}+\eta_{2}-2 \zeta\right)}\right], \\
& \beta=\arccos \left[1+\frac{2 \zeta\left(\zeta-\eta_{1}-\eta_{2}\right)}{\eta_{1} \eta_{2}}\right]-\alpha,
\end{aligned}
$$

where $\eta_{1}$ and $\eta_{2}$ must be replaced by the corresponding values according to Eqs. (23) and (24).

Moreover, Eq. (27) shows that $\kappa_{1}$ and $\kappa_{2}$ are constants, so that the drop is formed by the intersection of two spherical caps whose radii of curvature are given by

$$
R_{1}=\frac{R_{d}}{\sin \alpha}, \quad R_{2}=\frac{R_{d}}{\sin \beta}
$$

The dimensionless volume contribution of each spherical cap is obtained as a function of $R_{d}$ and the corresponding angle $\alpha$ or $\beta$ as

$$
\begin{aligned}
& V_{1}=\frac{\pi}{6} R_{d}^{3} \tan \frac{\alpha}{2}\left(3+\tan ^{2} \frac{\alpha}{2}\right), \\
& V_{2}=\frac{\pi}{6} R_{d}^{3} \tan \frac{\beta}{2}\left(3+\tan ^{2} \frac{\beta}{2}\right) .
\end{aligned}
$$

Considering that $V_{1}+V_{2}=V=4 \pi / 3$, the drop radius is

$$
R_{d}=8^{1 / 3}\left[3\left(\tan \frac{\alpha}{2}+\tan \frac{\beta}{2}\right)+\left(\tan ^{3} \frac{\alpha}{2}+\tan ^{3} \frac{\beta}{2}\right)\right]^{-1 / 3} \text {. }
$$




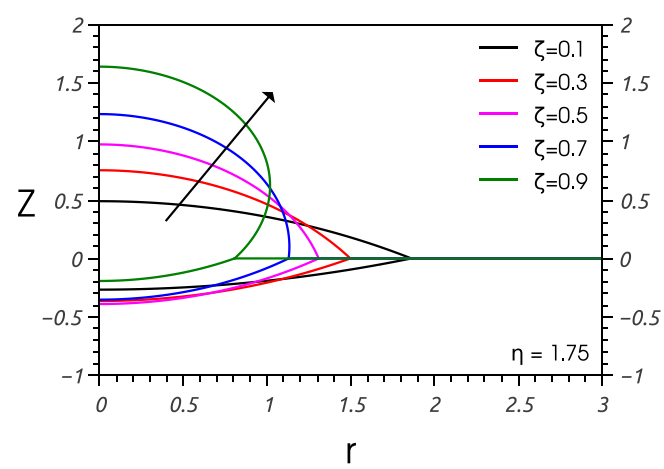

(a) Case A

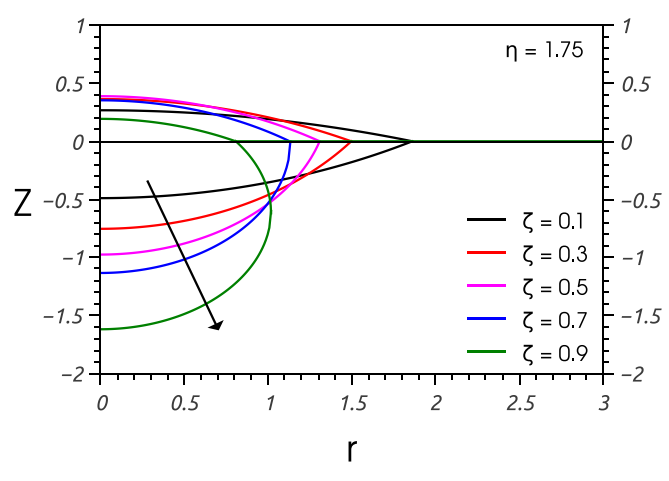

(b) Case B

FIG. 4. Solution without gravity: Drop profiles for $\eta=1.75$ and $\zeta=0.1,0.3,0.5,0.9$ for cases A $\left(\sigma_{1}<\right.$ $\left.\sigma_{2}\right)$ and $\mathrm{B}\left(\sigma_{1}>\sigma_{2}\right)$. Note that the free surface of the liquid substrate (curve 3 ) is flat for all $\zeta$.

In summary, for any value of $\sigma_{\text {ref }}$, Eqs. (28), (29), and (31) allow a calculation of the final equilibrium shapes for the entire range of possible values of $\eta$ and $\zeta$. For example, in Fig. 4 we show the drop profiles obtained for $\eta=1.75$ and $0.1<\zeta<1$ for case A [ $\sigma_{1}<\sigma_{2}$, Fig. 4(a)] and case B $\left[\sigma_{2}<\sigma_{1}\right.$, Fig. 4(b)]. The drop height $h_{d}$ increases in both cases A and B as $\zeta$ increases, mainly because of an $h_{t}$ (drop elevation) increase in case A and an $h_{b}$ (drop sinking) increase in case $\mathrm{B}$, as is schematically presented in Fig. 3. This effect is directly related to the spreading property of the drop given by the spreading factor $S$. In our present scheme, this feature is taken into account by the dimensionless parameter $\zeta$ [see Eq. (20)].

Interestingly, it can be shown that the lens shape of case A [Fig. 4(a)] corresponds to a reflection respect $z=0$ of case B [Fig. 4(b)], and vice versa. This is equivalent to changing the roles of fluids $\mathrm{B}$ and $\mathrm{C}$ (Fig. 2), as can be seen in Eqs. (15), where, by exchanging $\sigma_{1}$ and $\sigma_{2}, \theta_{A}$ does not change while both $\theta_{B}$ and $\theta_{C}$ change sign. In spite of this result, we still show here the two cases, $\mathrm{A}$ and $\mathrm{B}$, because the inclusion of gravity will break up this symmetry (see Sec. IV).

To further characterize the solution without gravity, we show in Fig. 5 the drop radius, $R_{d}$, as a function of $\zeta$ for $\eta=1.01,1.25,1.50,1.75$, and 2.00. We observe that for both cases, $R_{d}$ decreases

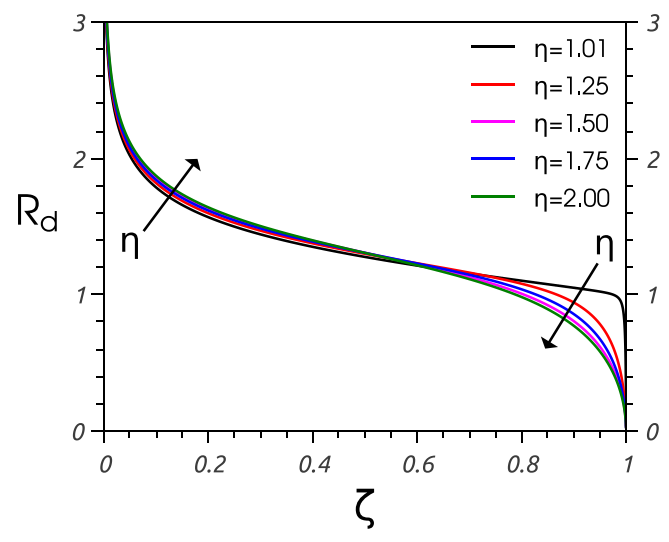

(a) Case A

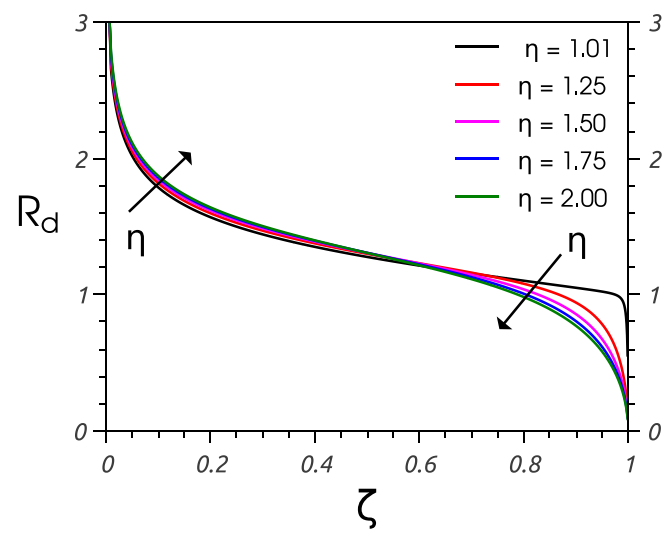

(b) Case B

FIG. 5. Solution without gravity: $R_{d}$ as function of $\zeta$ for $\eta=1.01,1.25,1.50,1.75$, and 2.00 for cases A $\left(\sigma_{1}<\sigma_{2}\right)$ and $\mathrm{B}\left(\sigma_{2}<\sigma_{1}\right)$. The arrows indicate the direction of increasing $\eta$. 


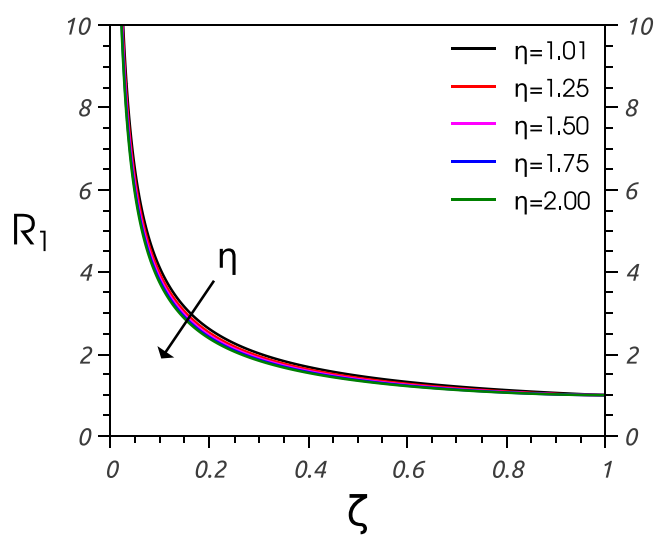

(a) Case A

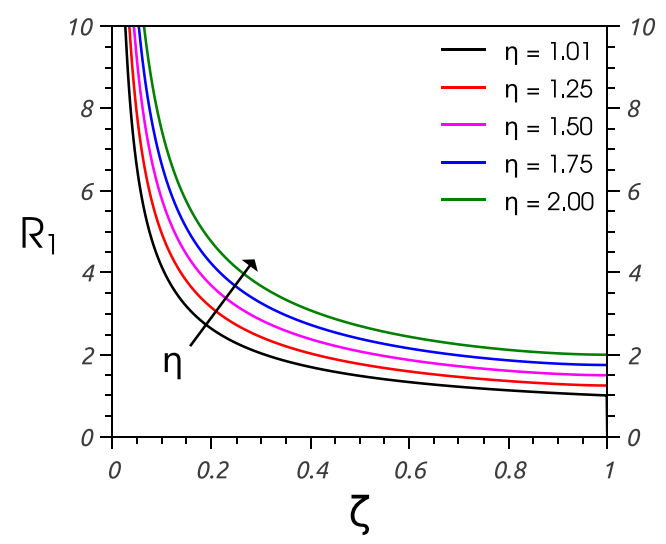

(c) Case B

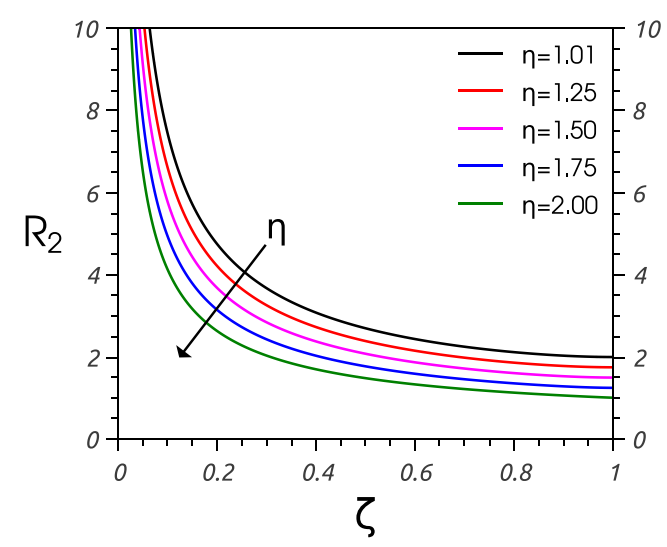

(b) Case A

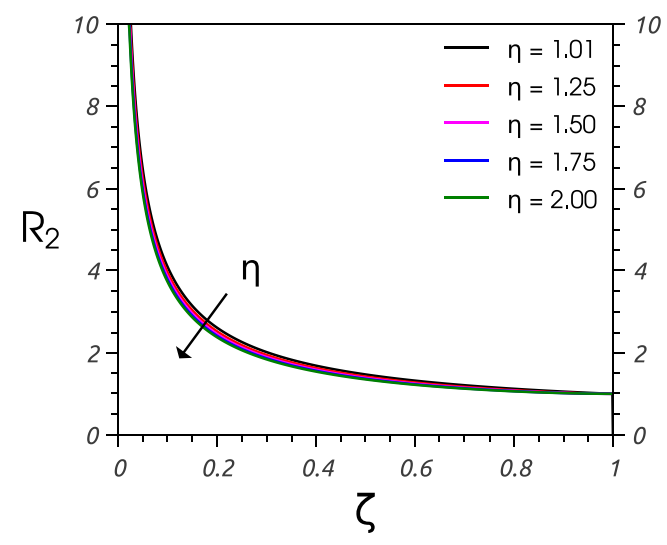

(d) Case B

FIG. 6. Solution without gravity: Radius of curvature of each surface of the lens as a function of $\zeta$ for $\eta=1.01,1.25,1.50,1.75$, and 2.00. (a) For curve 1 and (b) curve 2 in case A $\left(\sigma_{1}<\sigma_{2}\right)$, and (c) for curve 1 and (d) curve 2 in case $\mathrm{B}\left(\sigma_{2}<\sigma_{1}\right)$. The arrows indicate the direction of increasing $\eta$.

from large values at $\zeta \approx 0$ (which is near a complete wetting scenario) to $R_{d}=0$ at $\zeta=1$ [which corresponds to the nonwetting case imposed by the restriction in Eq. (19)]. The $\eta$-dependence is only significant for $\zeta \gtrsim 0.8$ and for $\eta \approx 1$. In this region, the combination of $\eta$ in its lower limit $(\eta \rightarrow 1)$ with $\zeta$ in its higher limit $(\zeta \rightarrow 1)$ implies $\eta_{3} \rightarrow 0$ [see Eqs. (21)-(24)], and consequently $\sigma_{3} \rightarrow 0$. Thus, this is the limit of miscibility of fluids B and C.

The particular shape of the drop is defined by the radius of curvature of each spherical cap. In Fig. 6 we show the radii $R_{1}$ of curve 1 and $R_{2}$ of curve 2 for $1<\eta<2$ and $0<\zeta<1$. In both cases these radii rapidly increase for $\zeta \rightarrow 0$ and approach a relatively flat zone as $\zeta \rightarrow 1$. We observe that $R_{1}$ in case $\mathrm{A}$ and $R_{2}$ in case $\mathrm{B}$ have the same behavior, while $R_{2}$ in case $\mathrm{A}$ and $R_{1}$ in case $\mathrm{B}$ also share an analogous behavior with the only difference that the dependence on $\eta$ occurs in opposite directions. The reason why $R_{1}$ in Case $\mathrm{A}$, as well as $R_{2}$ in Case $\mathrm{B}$ reach 1 at $\zeta=1$, is that the drop becomes completely spherical [see definition of the length scale in Eq. (1) and Fig. 3].

\section{TWO POSSIBLE SOLUTIONS WITH GRAVITY}

Unfortunately, it appears impossible to find an analytical solution of this three-phase problem with gravity. Therefore, we resort to the numerical solution of Eqs. (25) with the corresponding 


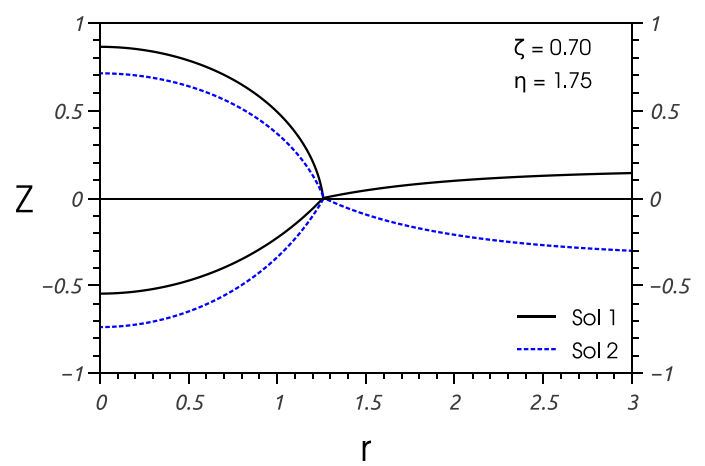

(a) Case A

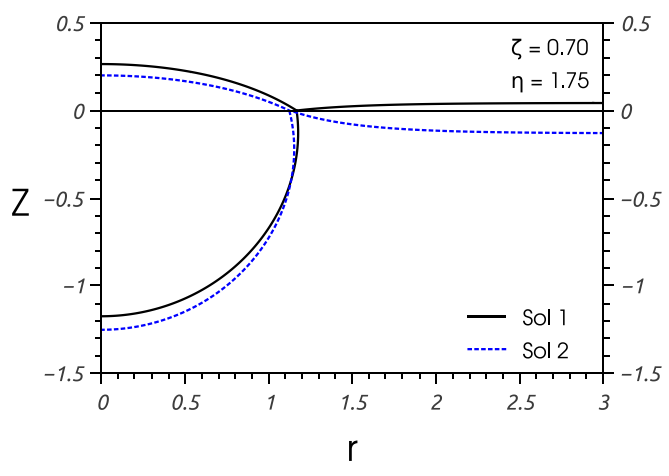

(b) Case B

FIG. 7. Example of the two families of solutions with gravity for $\eta=1.75$ and $\zeta=0.70$. (a) Case A $\left(\sigma_{1}<\sigma_{2}\right)$ and (b) Case B $\left(\sigma_{2}<\sigma_{1}\right)$.

dimensionless form of the conditions [Eqs. (10)-(14)]. To perform this task, we develop an iterative scheme based on seven dimensionless variables, namely $\left(R_{d}, L_{1}, L_{2}, L_{3}, P_{A}, P_{B}, P_{C}\right)$, and we fix the length scale by choosing a volume $\mathcal{V}_{0}$ [see Eq. (1)]. To define the values of the reference Bond number, Bo, and the density factors in Eqs. (25), we choose $\rho_{A}=0.97 \mathrm{~g} / \mathrm{cm}^{3}, \rho_{B}=1.0 \mathrm{~g} / \mathrm{cm}^{3}$, $\rho_{C}=0.0013 \mathrm{~g} / \mathrm{cm}^{3}$, and $\mathcal{V}_{0}=0.02 \mathrm{~cm}^{3}$. With these values, we have $\mathrm{Bo}=1.22$ and 0.49 for cases $\mathrm{A}$ and $\mathrm{B}$, respectively. We also choose $R_{\text {wall }}=2 \mathrm{~cm} / \mathcal{R}_{0}=11.88$.

To begin with, a first guess for $\left(L_{1}, L_{2}, L_{3}, R_{d}\right)$ can be taken from the analytical solution without gravity as obtained in Sec. III. Thus, $R_{d}^{(0)}$ is given by Eq. (31), and

$$
L_{1}^{(0)}=R_{1} \alpha, \quad L_{2}^{(0)}=R_{2} \beta, \quad L_{3}^{(0)}=R_{\text {wall }}-R_{d}^{(0)},
$$

where $(\alpha, \beta)$ and $\left(R_{1}, R_{2}\right)$ are given by Eqs. (28) and (29), respectively.

Since the solution without gravity does not contain reference pressures, we have no available values to guess for $\left(P_{A}, P_{B}, P_{C}\right)$. Here, we assume that $P_{A}^{(0)}$ and $P_{B}^{(0)}$ should be order 1 and of different signs because of the different orientation of the curvatures of curves 1 and 2 . Also, $P_{C}^{(0)}$ should be close to zero because we consider air as the surrounding fluid $\left(\rho_{C} \approx 0\right)$. However, the signs of these variables cannot be guessed for given $\eta$ and $\zeta$ based on any plausible argument. We find that any choice of these variables can lead to one of these three possibilities, namely the solution does not converge or it may converge to two different types of solutions. An example of them is shown in Fig. 7 for $\eta=1.75, \zeta=0.70$, and case A [Fig. 7(a)] or case B [Fig. 7(b)]. In Appendix we show that slight differences in the initial values of the parameters (mainly in the sign of $\Delta P_{3}$ ) could lead to any of these two possibilities. The existence of these two families, which we refer to as Sol 1 and Sol 2, do not depend on the choice of $\sigma_{\text {ref }}=\min \left(\sigma_{1}, \sigma_{2}\right)$, since we find that these two families exist for $\sigma_{1}<\sigma_{2}$ as well as for $\sigma_{1}>\sigma_{2}$.

For case A, the definitions in Eq. (23) hold. In Fig. 8 we show examples of the two families of solutions for $\eta=1.75$ and several $\zeta$ 's. The first family [Sol 1 in Fig. 8(a)] shows that the triple contact point is always under curve 3, while in the second family [Sol 2 in Fig. 8(b)] it is always above. Also, the shape of curve 3 near the triple contact point has a different sign of the curvature for each type of solution. In both cases, the drop radius decreases as $\zeta$ increases. It is interesting to note that as $\zeta$ increases, curve 3 increases its curvature.

Case B requires the use of the definitions in Eq. (24). In Fig. 9 we show examples of the two possible solution families for $\eta=1.75$ and several values of $\zeta$. As before, the drop radius decreases as $\zeta$ increases in both cases. However, there are few differences: in this case it is possible to have $\beta$ larger than $\pi / 2$, and both families converge to only one solution as $\zeta$ increases. Since the differences 


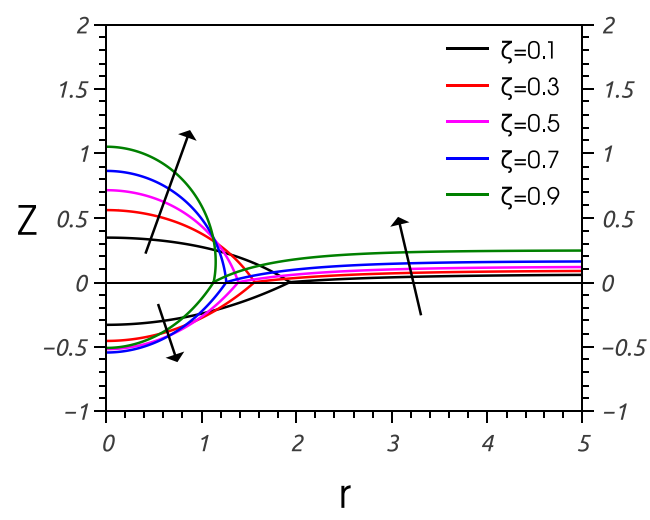

(a) Sol. 1

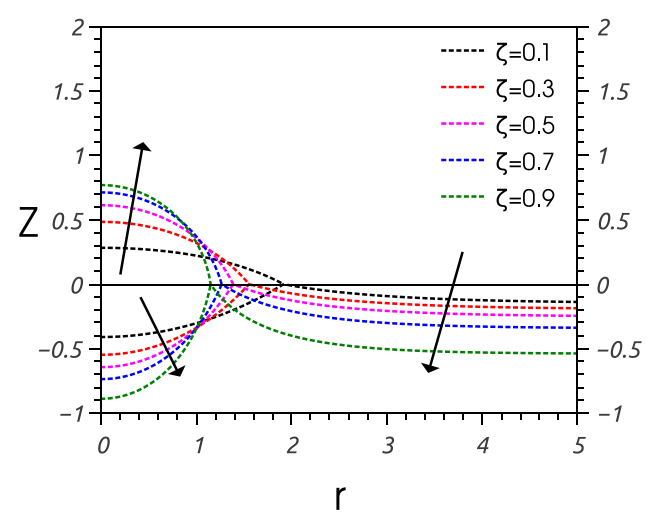

(b) Sol. 2

FIG. 8. Case A: Two families of solutions with gravity for $\eta=1.75$ and several values of $\zeta$. (a) Solutions type 1. (b) Solutions type 2. The arrows indicate the direction of increasing $\zeta$.

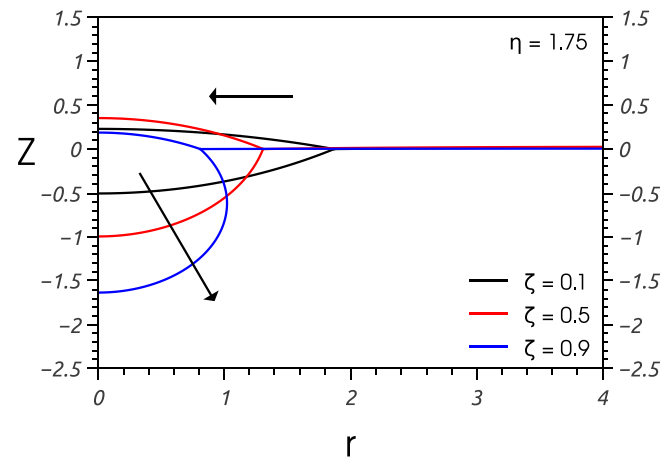

(a) Sol. $1(B o=0.49)$

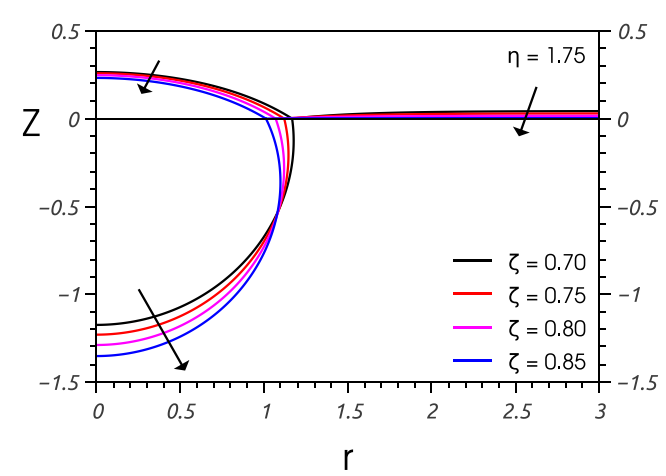

(c) Sol. $1(B o=4.32)$

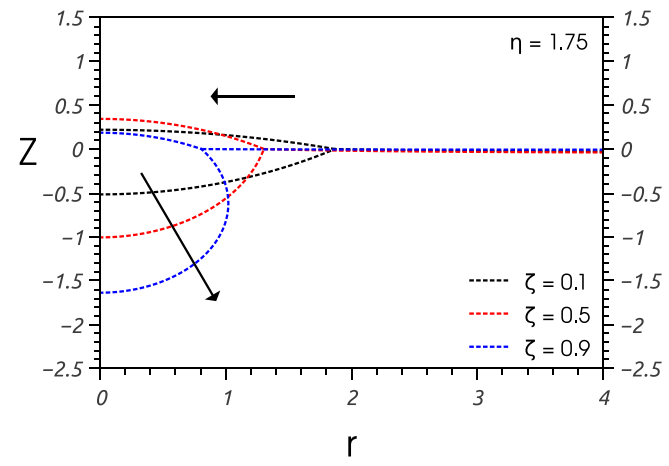

(b) Sol. $2(B o=0.49)$

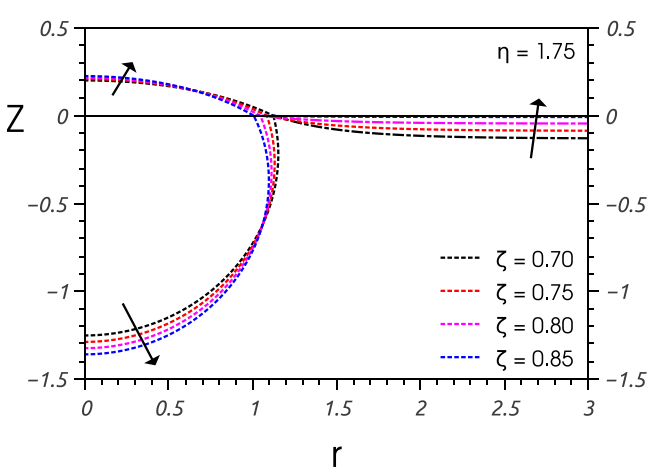

(d) Sol. $2(B o=4.32)$

FIG. 9. Case B: Two families of solutions with gravity for $\eta=1.75$ and several values of $\zeta$. (a) Solutions type $1(\mathrm{Bo}=0.49)$. (b) Solutions type $2(\mathrm{Bo}=0.49)$. (c) Solutions type $1(\mathrm{Bo}=4.32)$. (d) Solutions type 2 $(\mathrm{Bo}=4.32)$. The arrows indicate the direction of increasing $\zeta$. 


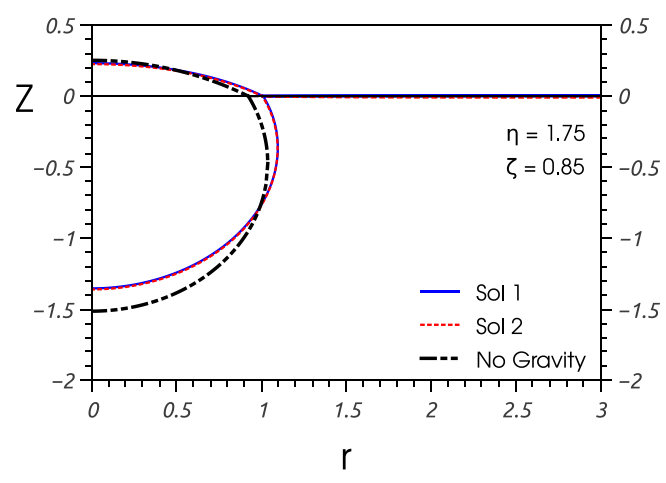

(a)

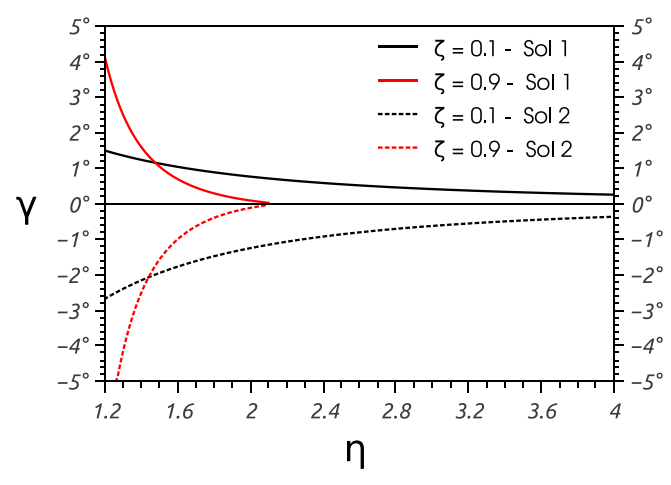

(b)

FIG. 10. Case B: (a) Two families of solutions with gravity for $\eta=1.75, \zeta=0.85$, and Bo $=4.32$ (practically coincident) compared with the analytical solution without gravity. (b) Evolution of angle $\gamma$ as a function of $\eta$ for both Sol 1 and Sol 2 for two extreme values of $\zeta$ and Bo $=0.49$. As $\eta$ increases, $\gamma$ decreases toward zero. The convergence to a flat Curve 3 is faster for larger $\zeta$.

between Sol 1 and 2 are not clearly visible in Figs. 9(a) and 9(b), we show them also for a larger volume in Figs. 9(c) and 9(d), where $\mathcal{V}_{0}=0.5 \mathrm{~cm}^{3}(\mathrm{Bo}=4.32)$.

Moreover, we observe a limiting solution for both Sol 1 and 2 at a certain pair of values $(\zeta, \eta)$ for which the curve 3 is practically flat, where both solutions become coincident. For the specific case in Figs. 9(c) and 9(d), these coincident solutions are shown in Fig. 10(a), where they are practically indistinguishable from one another (the solution without gravity for the same $\eta$ and $\zeta$ is also shown for comparison). In this case, the corresponding pair is $\zeta \approx 0.85$ and $\eta=1.75$. Note that the three solutions have a flat curve 3 and $\beta>\pi / 2$, while the no-gravity solution has smaller $R_{d}$ and larger $h_{d}$ than the gravitational solutions.

Note also that this convergence of solutions toward a single solution with flat curve 3 corresponds to $\gamma \rightarrow 0$. The behavior of this angle can be used as a probe to observe the differences between Sol 1 and Sol 2. In fact, in Fig. 10(b) we plot $\gamma$ as a function of $\eta$ for two extreme values of $\zeta$ as obtained for Solutions 1 and 2 for Bo $=0.49$. Clearly, their convergence occurs faster for larger $\zeta$ and smaller $\eta$.

Unfortunately, we do not have mathematical or physical arguments to explain why there are two different converged solutions for the same physical parameters. Clearly, this topic deserves further study, but it is beyond the scope of the present work. Therefore, we proceed by calculating the total energy of each family under the expectation that the one with lower energy is actually the one to be observed in nature.

\section{ENERGY ANALYSIS}

To analyze which of the equilibrium solutions identified in the previous section has lower energy, we calculate the total energy of each family of solutions for the problem with gravity as a function of $\eta$ and $\zeta$ for a fixed drop volume of $\mathcal{V}=0.2 \mathrm{~cm}^{3}$ and the corresponding Bo. We consider a region of finite size (i.e., a vessel, like in an experimental situation) in order to have finite values of the total energy, where we include both surface and volumetric (gravity) contributions. Then, we calculate the difference between the total energy of the solution, $E$, and that of the system without the drop in it, $E_{0}$.

The integration domain is depicted in Fig. 11, which is a cylindrical container of radius $R_{\text {wall }}$ and height $H$. The level of fluid $B$ changes from $h_{f}$ to $h_{f}^{*}$ as the drop is deposited on its surface, since no flow is allowed through the vessel walls $\left(V_{B}=\right.$ const $)$. The total dimensional energy is the sum 


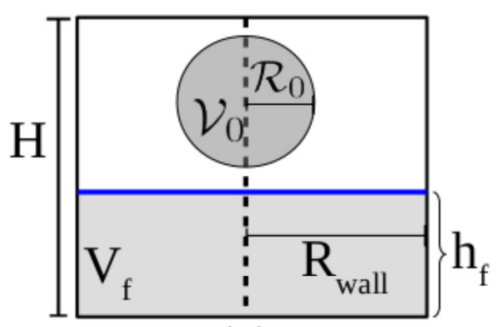

(a)

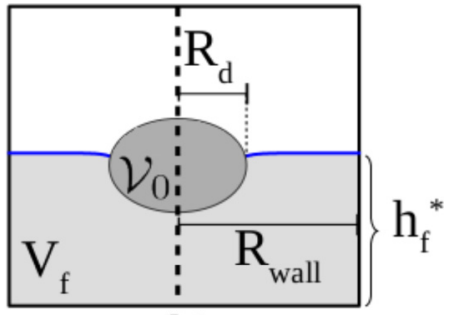

(b)

FIG. 11. (a) Initial and (b) final states of the approach used to calculate the system energy: it corresponds to a finite volume configuration with fixed $V_{f}$ and variable $h_{f}$.

of the surface, $E_{S}$, and gravitational energy, $E_{V}$. Initially, we have only fluid $B$ in the vessel, so that

$$
E_{0}=E_{S, 0}+E_{V, 0}=\sigma_{3} \pi \mathcal{R}_{0}^{2} R_{\mathrm{wall}}^{2}+\frac{\pi}{2} \rho_{B} g \mathcal{R}_{0}^{4} h_{f}^{2} R_{\mathrm{wall}}^{2},
$$

since we neglect the gravitational energy of fluid $C$ (air). To calculate the final energy,

$$
E=E_{S}+E_{V}
$$

we consider the surface contributions of the three interfaces plus the gravitational contributions for liquids $A$ and $B$ :

$$
\begin{aligned}
E_{S}= & 2 \pi \mathcal{R}_{0}^{2}\left(\sigma_{1} L_{1} \int_{0}^{1} r_{1}(q) d q+\sigma_{2} L_{2} \int_{0}^{1} r_{2}(q) d q+\sigma_{3} L_{3} \int_{0}^{1} r_{3}(q) d q\right), \\
E_{V}= & \pi \rho_{A} g \mathcal{R}_{0}^{4}\left(\int_{0}^{1}\left[z_{1}^{2}(q)-z_{\mathrm{tp}}^{2}\right] r_{1}(q) r_{1}^{\prime}(q) d q+\int_{0}^{1}\left[z_{\mathrm{tp}}^{2}-z_{2}^{2}(q)\right] r_{2}(q) r_{2}^{\prime}(q) d q\right) \\
& +\pi \rho_{B} g \mathcal{R}_{0}^{4}\left(\int_{0}^{1} z_{2}^{2}(q) r_{2}(q) r_{2}^{\prime}(q) d q+\int_{0}^{1} z_{3} r_{3}(q) r_{3}^{\prime}(q) d q\right),
\end{aligned}
$$

where the integrals inside the parentheses are dimensionless quantities. Here, $z_{\text {tp }}$ is the $z$-coordinate of the triple point and $z=0$ corresponds to the bottom of the control region. The thickness $h_{f}$ is large enough to assure that the drops do not touch the solid substrate for all of the calculations.

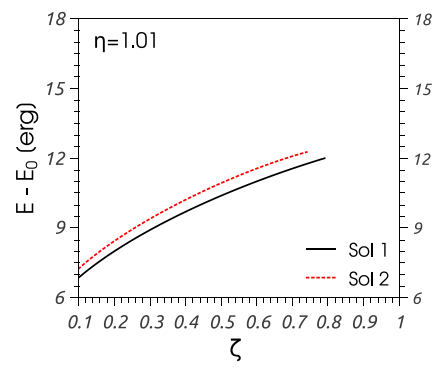

(a)

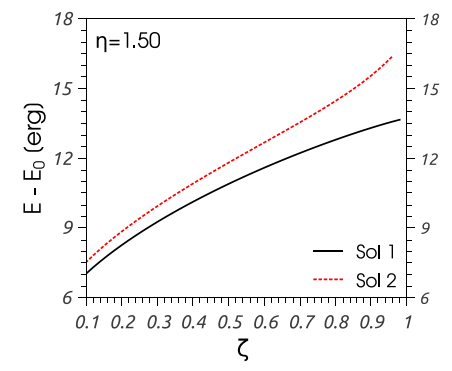

(b)

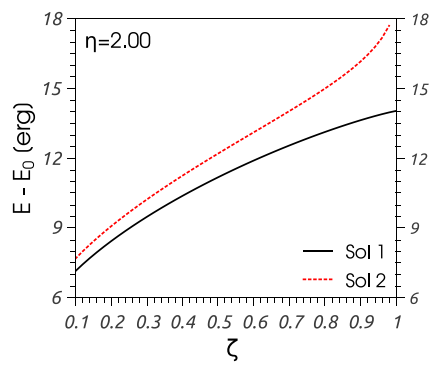

(c)

FIG. 12. Case A: Total energy variation for Bo $=1.22$, Sol 1 (solid lines) and Sol 2 (dashed lines). (a) $\eta=1.01$, (b) $\eta=1.5$, (c) $\eta=2$. The curves in (a) do not go beyond $\zeta \approx 0.8$ because of precision issues in the numerical scheme appearing when both $\xi$ and $\eta$ are very close to unity (their respective maximum and minimum limiting values). 


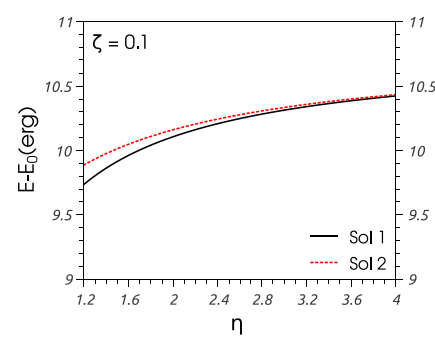

(a)

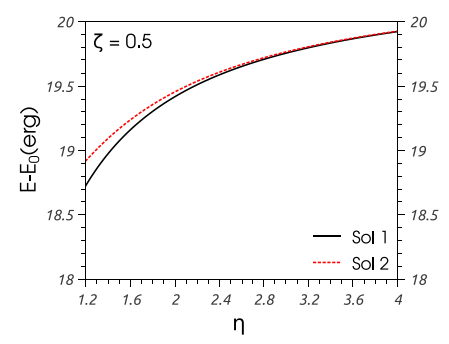

(b)

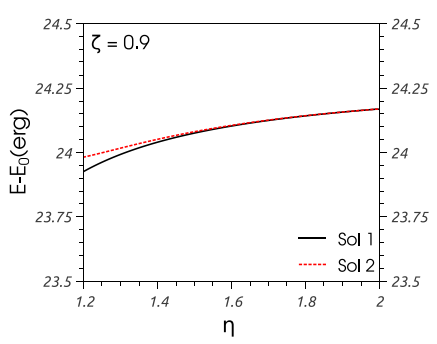

(c)

FIG. 13. Case B: Total energy variation for $\mathrm{Bo}=0.49$, Sol 1 (solid lines) and Sol 2 (dashed lines). (a) $\zeta=$ 0.1, (b) $\zeta=0.5$, (c) $\zeta=0.9$.

The variation of the total energy for the two families of solutions is shown for case A in Fig. 12. We find that Sol 1 always has lower energy than Sol 2 for different combinations of $(\eta, \zeta)$. The energy difference between the two solutions increases as both $\eta$ and $\zeta$ increase.

On the other hand, for case B, we find the curves shown in Fig. 13. In this case, Sol 1 also remains with lower energy than Sol 2 for $\eta \approx 1$, but their difference decreases as $\eta$ increases. This decrease occurs faster as $\zeta$ is closer to unity. This is consistent with the result that both solutions tend to converge to a single solution in this case. In summary, the energy analysis shows that Sol 1 is more likely to occur in nature for both cases A and B, since it is always lower in energy.

With the aim to check that our theoretical results point in the right direction, we perform a preliminary experiment where we place a silicon oil [polydimethylsiloxane (PDMS)] drop on top of a water surface. For this configuration we have $\eta=1.79$ and $\zeta=0.04$, so that it corresponds to a

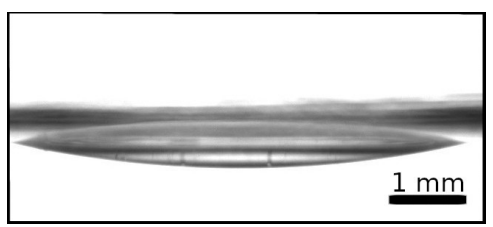

(a)

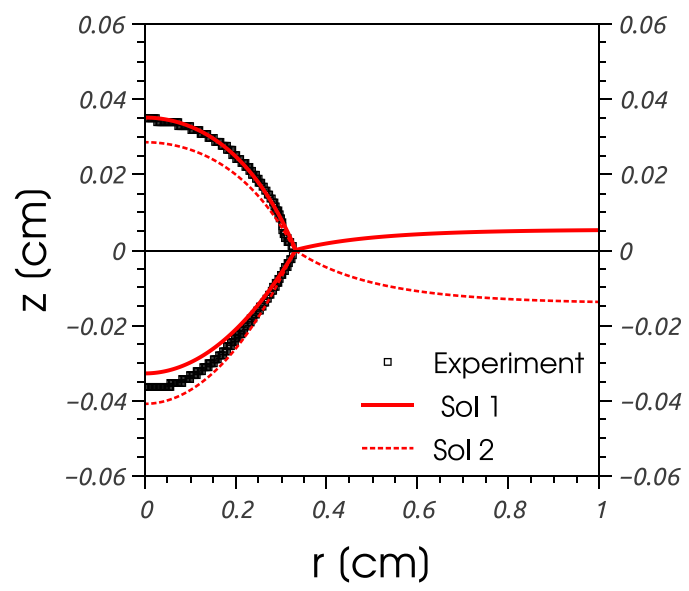

(b)

FIG. 14. (a) Experiment: Silicon oil (PDMS) drop on water (case A with $\eta=1.79$ and $\zeta=0.04$ ). (b) Comparison of drop shape with solutions Sol 1 and Sol 2. 


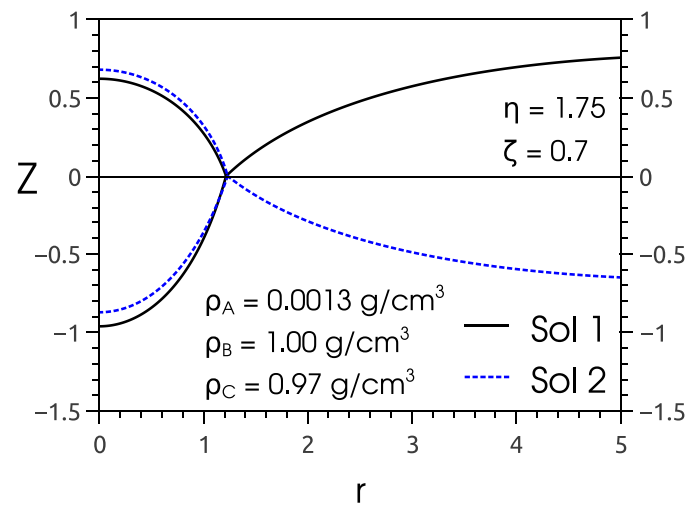

FIG. 15. Air bubble trapped between two dense immiscible liquids with gravity: $\rho_{A}=0.0013 \mathrm{~g} / \mathrm{cm}^{3}$ (air), $\rho_{B}=1.00 \mathrm{~g} / \mathrm{cm}^{3}$ (water), and $\rho_{C}=0.97 \mathrm{~g} / \mathrm{cm}^{3}$ (oil). Here, Sol 1 is the black solid line, while Sol 2 is the blue dashed line.

case near the limit of total spreading [see Fig. 14(a)]. Unfortunately, we are not yet able to optically obtain an image of curve 3, i.e., the water/air interface. Nevertheless, the comparison of the shapes of both curve 1 (oil/air interface) and curve 2 (oil/water interface) with the solutions shows that Sol 1 is closer to the lens shape than Sol 2 [see Fig. 14(b)]. This encouraging comparison with an experiment supports our conjecture that Sol 1 is most probably found in nature. A more detailed experimental study is left for future work.

Finally, we briefly investigate the case in which $\rho_{A}<\rho_{C}<\rho_{B}$, which could correspond to a bubble trapped between two immiscible liquids. Figure 15 shows that also in this case there exist two families of solutions with similar characteristics to those seen in previous cases (note that we use now the same values of $\eta$ and $\zeta$ as in Fig. 7). In this scenario, the energy of Sol 2 is smaller than that of Sol 1 by $\approx 30 \%$. Therefore, unlike the case of heavy drops such as that in Fig. 7, Sol. 2 is more probably found in nature. This result suggests a reasonable buoyant behavior of a bubble when surrounded by more dense media.

\section{SUMMARY AND CONCLUSIONS}

Although there are many physical parameters involved in the determination of the shape of a liquid lens, we present here a dimensionless scheme that embraces all possible physical situations. The use of the parameters $\eta$ and $\zeta$ [see Eqs. (20)-(22)] allows us to describe the behavior of the solutions for any combination of the three surface tensions $\sigma_{1}, \sigma_{2}$, and $\sigma_{3}$.

Within this framework, we have analytically solved the case without gravity and numerically analyzed the case with gravity. The no-gravity solution is presented as a tool to obtain the initial guesses for the numerical analysis performed in the more general case with gravity. A remarkable fact, not usually mentioned in the literature, is the requirement that the spreading factor $S$ is bounded from below, i.e., $S^{*}<S<0$, for equilibrium solutions to exist. This implies $0<\zeta<1$, which is more restrictive than the usual case for liquid drops on solids.

We have highlighted that the effects of gravity on the solution are far from trivial. For example, it is possible to obtain two different families of solutions for the same set of physical parameters. Although some of these solutions have been reported in the literature, this issue has apparently been unrecognized because the authors have assumed that, regardless of the set of guess values, any converged solution is valid based on the belief that it is unique. However, we show here that the solutions can be nonunique, and hence a more rigorous treatment is needed. As mentioned before, the proof of uniqueness of the solution for the mathematical problem posed by Eqs. (10)-(14) is beyond the scope of the present work. The main difference between the solution families is the 
shape of the free surface of the liquid $B$ near the lens, where the curvature of curve 3 (see Fig. 7) adopts a different sign for each family.

To decide which solution is more likely to be found in nature, we also perform an energy analysis to compare the two families of solutions under two possible scenarios, namely case $\mathrm{A}\left(\sigma_{1}<\sigma_{2}\right)$ or case $\mathrm{B}\left(\sigma_{1}>\sigma_{2}\right)$. This analysis is done considering a finite volume of liquid $B$ (so it is contained in a vessel). It turns out that in both scenarios Sol 1 is always less energetic than Sol 2, so that it is most likely to be found in natural situations. Moreover, it is found that the two solution families converge to a unique one when $\sigma_{1}>\sigma_{2}$, and both $\eta$ and $\zeta$ are large enough.

\section{ACKNOWLEDGMENTS}

The authors gratefully acknowledge suggestions made by the referees. P.D.R., A.G.G., and J.A.D. acknowledge support from Consejo Nacional de Investigaciones Científicas y Técnicas (CONICET, Argentina) and Agencia Nacional de Promoción Científica y Tecnológica (ANPCyT, Argentina) with Grant PICT 1067/2016. H.A.S. research was partially supported by NSF through Princeton University's Materials Research Science and Engineering Center DMR-1420541.

\section{APPENDIX: INITIAL CONFIGURATION OF THE NUMERICAL PROCEDURE}

As mentioned in Sec. IV, three possibilities arise from the choice of the guess values in the numerical scheme: no convergence, or convergence to Sol 1 or Sol 2 families. These two kinds of solutions were found before (see Fig. 4 in [12]), but with different sets of parameters. So, one set led to a solution with concave curvature of surface 3 and the other one with convex curvature. Thus, it was not possible to assert that they belonged to different families, because of the belief that the solution is unique. Therefore, there was no mention on the possibility of finding them for the same set of physical parameters.

To clarify this issue, we perform a detailed analysis on the selection of the guess values for the iteration procedure for the case with $\rho_{A}=0.7 \mathrm{~g} / \mathrm{cm}^{3}, \rho_{B}=1.0 \mathrm{~g} / \mathrm{cm}^{3}, \sigma_{1}=25 \mathrm{mN} / \mathrm{m}, \sigma_{2}=$ $55 \mathrm{mN} / \mathrm{m}, \sigma_{3}=70 \mathrm{mN} / \mathrm{m}$, and $\mathcal{V}_{0}=0.579 \mathrm{~cm}^{3}$, as presented in Fig. 4(a) in [12].

First, we calculate the corresponding dimensionless parameters $\eta=2.2$ and $\zeta=0.2$, as well as the length scale given by Eq. (1). Then, we follow the procedure described in Sec. III to analytically obtain the solution without gravity. Within this framework, we find $R_{d}=$ $1.64811, L_{1}=1.81602, L_{2}=1.66852, R_{1}=2.35493, R_{2}=5.18084, \alpha=44.4^{\circ}$, and $\beta=18.5^{\circ}$. With these results and $L_{3}^{(0)}=R_{\text {wall }}-R_{d}^{(0)}$, we can construct two sets of guess values for $G=$ $\left(\Delta P_{1}^{(0)}, \Delta P_{2}^{(0)}, \Delta P_{3}^{(0)}, L_{1}^{(0)}, L_{2}^{(0)}, L_{3}^{(0)}, R_{d}^{(0)}\right)$, namely

$$
\begin{aligned}
& G_{1}=(-0.2,0.5,+0.0001,1.81602,1.66852,4.15407,1.64811), \\
& G_{2}=(-0.2,0.5,-0.0001,1.81602,1.66852,4.15407,1.64811),
\end{aligned}
$$

where $\Delta P_{1}^{(0)}$ and $\Delta P_{2}^{(0)}$ were chosen order 1 with different signs between each other, and $\Delta P_{3}^{(0)} \sim$ $10^{-4}$ has a different sign in each set of parameters. This slight difference in the initial values leads to the two solution families. The surface profiles obtained with these sets of guess values are shown in Fig. 16. The resulting values for the solution sets $T=\left(\Delta P_{1}, \Delta P_{2}, \Delta P_{3}, L_{1}, L_{2}, L_{3}, R_{d}\right)$ are

$$
\begin{aligned}
& T_{1}=(-0.05404,0.23077,+0.00012,1.97932,1.97290,3.86900,1.93438) \\
& T_{2}=(-0.00888,0.57527,-0.00049,1.73873,1.95947,4.12058,1.73836)
\end{aligned}
$$

According to our analysis in Sec. V, the Sol 2 family corresponds to a higher energy case, so it is not likely to be found in nature. Fortunately, Sol 1 was correctly reported in [12]. 


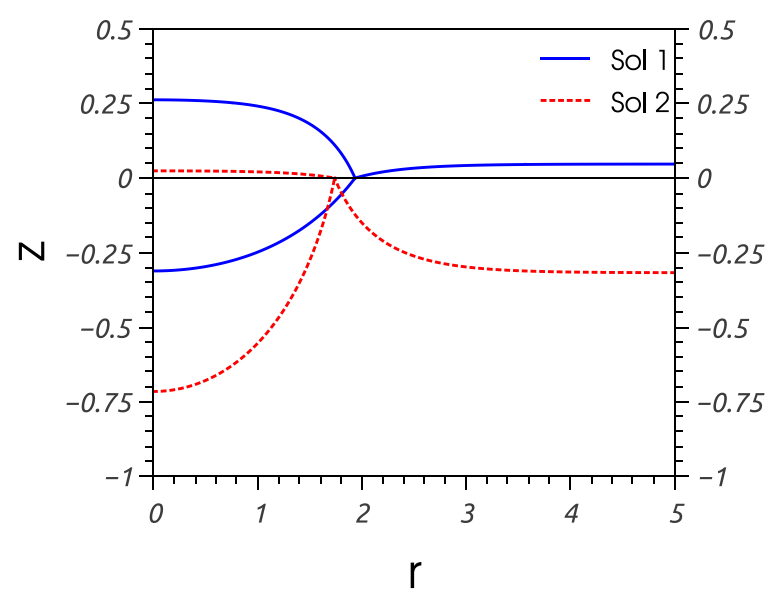

FIG. 16. Sol 1 (blue, solid) reported in Fig. 4(a) in [12], and Sol 2 (red, dashed) the other possible solution obtained for the same set of physical parameters (see the text).

[1] B. Franklin, W. Brownrigg, and N. Farish, Of the stilling of waves by means of oil. Extracted from sundry letters between Benjamin Franklin, William Brownrigg, and the Reverend Mr. Farish, Philos. Trans. R. Soc. London 64, 445 (1774).

[2] L. Rayleigh, On foam, Proc. R. Inst. G.B. 13, 85 (1890).

[3] F. E. Neumann and A. Wangerin, Vorlesung über die Theorie der Capillarität (Teubner, Leipzig, 1894).

[4] W. B. Hardy, The tension of composite fluid surfaces and the mechanical stability of films of fluid, Proc. R. Soc. London, Ser. A 86, 610 (1912).

[5] C. G. Lyons, The angles of floating lenses, J. Chem. Soc., 623 (1930).

[6] I. Langmuir, Oil lenses on water and the nature of monomolecular expanded films, J. Chem. Phys. 1, 756 (1933).

[7] N. A. Miller, Investigation of the tension mechanisms responsible for lens formation and a new method for measuring the angles of liquid lenses, J. Phys. Chem. 45, 1025 (1941).

[8] W. A. Zisman, The spreading of oils on water. Part I. Ionized molecules having only one polar group, J. Chem. Phys. 9, 534 (1941).

[9] Y. Seeto, J. E. Puig, L. E. Scriven, and H. T. Davis, Interfacial tensions in systems of three liquid phases, J. Colloid Interface Sci. 96, 360 (1983).

[10] K. Takamura, N. Loahardjo, W. Winoto, J. Buckley, N. R. Morrow, M. Kunieda, Y. Liang, and T. Matsuoka, Spreading and retraction of spilled crude oil on sea water, in Crude Oil Exploration in the World (IntechOpen, Rijeka, 2012), Chap. 6, pp. 107-124.

[11] F. B. Wyart, P. Martin, and C. Redon, Liquid/liquid dewetting, Langmuir 9, 3682 (1993).

[12] J. C. Burton, F. M. Huisman, P. Alison, D. Rogerson, and P. Taborek, Experimental and numerical investigation of the equilibrium geometry of liquid lenses, Langmuir 26, 15316 (2010).

[13] M. Tress, S. Karpitschka, P. Papadopoulos, J. H. Snoeijer, D. Vollmer, and H. Butt, Shape of a sessile drop on a flat surface covered with a liquid film, Soft Matter 13, 3760 (2017).

[14] P. Chen, S. S. Susnar, C. Mak, A. Amirfazli, and A. W. Neumann, Lens size dependence of contact angle and the line tension of the dodecane-water-air system, Colloids Surf. A 129-130, 45 (1997).

[15] J. McBride, J. Cary, and C. Simmons, Direct measurement of the spreading pressure of organic liquids on water, J. Colloid Interface Sci. 132, 363 (1989).

[16] K. Endoh, A. Mikami, and Y. Mori, Determination of the spreading coefficient of one liquid on another by means of interferometric measurements of liquid-lens thickness, Colloids Surf. 46, 99 (1990).

[17] V. G. Levich and V. S. Krylov, Surface-tension-driven phenomena, Annu. Rev. Fluid Mech. 1, 293 (1969). 
[18] J. Sebilleau, Equilibrium thickness of large liquid lenses spreading over another liquid surface, Langmuir 29, 12118 (2013).

[19] A. Nikolov and D. Wasan, Oil lenses on the air-water surface and the validity of Neumann's rule, Adv. Colloid Interface Sci. 244, 174 (2017).

[20] R. Iqbal, S. Dhiman, A. K. Sen, and A. Q. Shen, Dynamics of a water droplet over a sessile oil droplet: Compound droplets satisfying a Neumann condition, Langmuir 33, 5713 (2017).

[21] L. Liu, C. Xu, L. Zhao, M. Mi, and C. Li, Experimental and theoretical study of evaporation of a volatile liquid lens on an immiscible liquid surface, Langmuir 35, 12979 (2019).

[22] Q. He, D. Liu, W. Huang, and J. Wang, Interactions of oil drops induced by the lateral capillary force and surface tension gradients, Langmuir 35, 14967 (2019).

[23] T. V. Vinay and S. N. Varanakkottu, Separation of floating oil drops based on drop-liquid substrate interfacial tension, Langmuir 35, 10596 (2019).

[24] Y. Zhang, D. Chatain, S. L. Anna, and S. Garoff, Stability of a compound sessile drop at the axisymmetric configuration, J. Colloid Interface Sci. 462, 88 (2016).

[25] L. Mahdevan, M. Adda-Bedia, and Y. Pomeau, Four-phase merging in sessile compound drops, J. Fluid Mech. 451, 411 (2002).

[26] M. J. Neeson, R. F. Tabor, F. Grieser, R. R. Dagastine, and D. Y. C. Chan, Compound sessile drops, Soft Matter 8, 11042 (2012).

[27] S. Torza and S. Mason, Three-phase interactions in shear and electrical fields, J. Colloid Interface Sci. 33, 67 (1970).

[28] D. S. Ross, An algorithm for determining the shapes of floating fluid lenses, J. Colloid Interface Sci. 154, 66 (1992).

[29] A. Elcrat, R. Neel, and D. Siegel, Equilibrium configurations for a floating drop, J. Math. Fluid Mech. 6, 405 (2004).

[30] C. M. Phan, B. Allen, L. B. Peters, T. N. Le, and M. O. Tade, Can water float on oil? Langmuir 28, 4609 (2012).

[31] C. Y. H. Wong, M. Adda-Bedia, and D. Vella, Non-wetting drops at liquid interfaces: From liquid marbles to Leidenfrost drops, Soft Matter 13, 5250 (2017). 